\title{
Woods Hole Mathematics
}

Perspectives in Mathematics and Phussics 
This page is intentionally left blank 
REE Series on Knots and Everything - Vol. 34

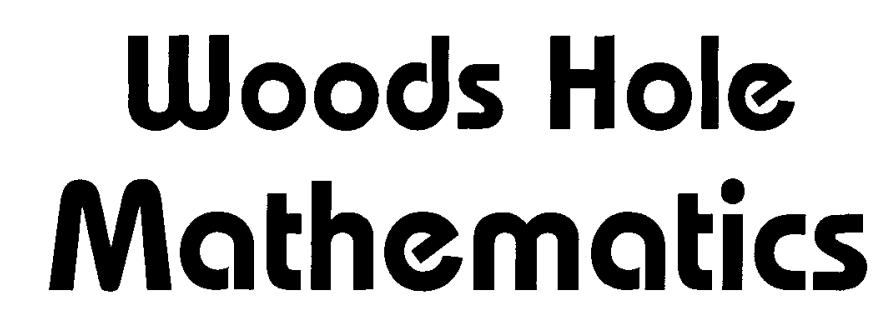

Perspectives in Mathematics and Phussics

editors

Nils Tongring

Woods Hole Oceonographic Institution, USA

R C Penner

University of Southern Colifornia, USA

\section{$\|=$ World Scientific}




\section{Published by}

World Scientific Publishing Co. Pte. Ltd.

5 Toh Tuck Link, Singapore 596224

USA office: 27 Warren Street, Suite 401-402, Hackensack, NJ 07601

UK office: 57 Shelton Street, Covent Garden, London WC2H 9HE

\section{Library of Congress Cataloging-in-Publication Data}

Woods Hole mathematics : perspectives in mathematics and physics / editors, Nils Tongring,

R. C. Penner.

p. cm. -- (K \& E series on knots and everything ; v. 34)

Includes bibliographical references.

ISBN 9812560211 (alk. paper)

1. Mathematical physics. 2. Operads. 3. Quantum algebra. 4. Differential equations.

5. Quantum physics. 6. Moduli theory. I. Tongring, Nils. II. Penner, R. C., 1956- III. Title.

IV. Series.

QC20.W665 2005

$530 \cdot 15--\mathrm{dc} 22$

2004059531

\section{British Library Cataloguing-in-Publication Data}

A catalogue record for this book is available from the British Library.

\section{Copyright 12004 by World Scientific Publishing Co. Pte. Ltd.}

All rights reserved. This book, or parts thereof, may not be reproduced in any form or by any means, electronic or mechanical, including photocopying, recording or any information storage and retrieval system now known or to be invented, without written permission from the Publisher.

For photocopying of material in this volume, please pay a copying fee through the Copyright Clearance Center, Inc., 222 Rosewood Drive, Danvers, MA 01923, USA. In this case permission to photocopy is not required from the publisher. 


\section{PREFACE}

The following eight papers are by mathematicians and physicists connected in some way to the three meetings held at the Woods Hole Oceanographic Institution (WHOI) during the past six years. This book is not a proceedings in the usual sense, but expositions of recent work contributed by those researchers interested in this form, and written with no pressure from editors. The topics of the meetings were suggested by the interests of the Sullivan Seminar at the City University of New York Graduate Center from diverse areas of mathematics and physics, but related certainly in concepts and techniques. One of the strands of this mix may have started in Woods Hole forty years ago (cf. the reminiscences of Raoul Bott in the Introduction to this book).

The first meeting (1998) at Woods Hole was on fluid flow: the perennial problems of the Euler and Navier-Stokes equations and turbulence. A main theme was alternatives to the continuum model as a way of overcoming issues of regularity, the combinatorial difficulty of completing the evolution model when shock waves develop, and the limits to any numerical computation. When the continuum equations are written in finite terms, a fundamental algebraic problem appears: the symmetry of the infinitesimal algebra is broken. From this point Sullivan took the first steps towards a combinational geometry in fluid flow, later echoed in his work on string topology.

The 1999 meeting celebrated the 35th anniversary of the Woods Hole (or Atiyah-Bott) theorem, the eponym reflecting the cooperative nature of the original proof (cf. Bott, ibid). The event was one of many marking the end of Bott's formal teaching career at Harvard. The central topic of the meeting was the integral and combinatorial invariants of three-manifolds.

The third meeting (2001), Graph Theory: Confluences in Molecular Biology and the Physical Sciences, studied the uses of diagram techniques in molecular biology, physics, and Teichmüller theory. Lenore Cowen gave an example of using both proteins to isolate a feature of secondary structure that may signal disease. Both Louis Kauffman and Bob Penner gave possible applications of their work to molecular biology (cf. their contributions in this volume).

Below is a list of the speakers from the last three meetings; the list might be compared with the list of participants in the 1964 meeting given by Bott in the Introduction: 

S. Axelrod
C. Epstein
R. Kaufman
T. Tangerman
R. Bott
M. Farge
G. Kuperberg
D. Thurston
L. Chekhov
K. Helfrich
S. Martin
J. Weitsman
L. Cowen
L. Kauffman
R. Penner

Our participants were of course a much larger group, whose names might be difficult to recover; there were nine days of meetings, not a month. But the summer of 1964 was a more leisurely time, and rambling houses on Quissett Harbor were still within the reach of academics.

The meetings at Woods Hole were possible only through the support of WHOI Senior Scientist Andrew M. Solow, the institution itself, and the National Science Foundation.

The Marine Biological Laboratory in Woods Hole has been generous in providing lecture rooms and housing when they were needed.

Nils Tongring

Bob Penner

New York

Los Angeles

May 1, 2004 


\section{INTRODUCTION}

Woods Hole has played such a vital role in both my mathematical and personal life that it is a great pleasure to see the mathematical tradition of the 1964 meeting resurrected forty years later and, as this volume shows, resurrected with new vigor and one hopes on a regular basis. I therefore consider it a signal honor to have been asked to introduce this volume with a few reminiscences of that meeting forty years ago.

The situation of Woods Hole is ideally suited for intellectual enterprises. On the one hand, it offers the spectacular scenery of an active harbor and beautiful access to the ocean, while on the other hand, it has few of the irritating distractions of a truly touristy place. I hope finally the facilities of the Woods Hole Oceanographic Institution, recently augmented with the beautiful Whitney estate, will continue as a conference center available to the mathematical community. Our thanks for the effort to bring this about are due to Nils Tongring and Andy Solow.

The 1964 meeting was of course at this same Whitney estate, and all our lectures took place in the rustic rooms of the main building. The participants were housed in various rented cottages, and I now recall with a great sense of nostalgia the large rambling house on the ocean front overlooking the Quissett harbor, which my family of six shared with the Tate family of four, and with Serre and his daughter. The Bott and Tate families had already spent a summer together harmoniously and the addition of the Serres now only added to the fun and excitement of this adventure in communal living.

Thanks to David Mumford's amazing filing habits, far exceeding those of the Mathematical Society, I can also reproduce here a hopefully complete list of the participants:

$\begin{array}{lllll}\text { Abhyankar } & \text { Ehrenpreis } & \text { Kulkarni } & \text { Rasala } & \text { Shimura } \\ \text { Artin } & \text { Greenleaf } & \text { Lichtenbaum } & \text { Rauch } & \text { Tate } \\ \text { Atiyah } & \text { Hartshorne } & \text { Lubin } & \text { Rim } & \text { Verdier } \\ \text { Auslander } & \text { Hironaka } & \text { Matsusaka } & \text { Rosenlicht } & \text { Waterhouse } \\ \text { Bott } & \text { Igusa } & \text { Mayer } & \text { Samuel } & \text { Zariski } \\ \text { Cassels } & \text { Kodaira } & \text { Mumford } & \text { Schlessinger } & \\ \text { Dwork } & \text { Kuga } & \text { Nagata } & \text { Serre } & \end{array}$

The conference was mainly concerned with questions of algebraic geometry and number theory, and much of what was going on was way above my head. However, my friend and collaborator Michael Atiyah and I soon 
found ourselves in hot pursuit of a version of the Lefschetz fixed point theorem, which was applicable to "elliptic complexes" in general, and therefore paid scant attention to other aspects of the conference. But the impetus for this work certainly came from some lectures in number theory, where we heard about the work of Eichler on Riemann surfaces, and thereafter Shimura explained his beautiful conjectured generalization of Eichler's formula to us. Actually I seem to recall that Atiyah and I stumbled on some version of this formula ourselves, but as no one else is able to confirm this fact, I now have to attribute it to the universal self-aggrandizing instincts of memory. In any case we were immediately smitten by Shimura's formula and hell-bent to prove it.

Our attack was two pronged. On the one hand we felt that in the presence of so many high-powered algebraic geometers, well versed in the principles of derived functors and internal homs, one should be able to coax a proof of the Shimura formula from the participants. So we organized a seminar devoted solely to this purpose and by the end of the conference our objective had been achieved. In view of the collaborative nature of the enterprise we dubbed it the Woods Hole fixed point theorem.

A very vivid memory is the walk in the grounds of the estate during which Michael and I first realized that Hermann Weyl's character formula was a surprisingly simple and natural consequence of this fixed point formula. Those interested in an account of these developments, as well as other aspects of the conference, can find an excellent one in the recently published bilingual edition of the correspondence of Serre and Grothendieck.

On the other hand we worked on the generalization of this formula to arbitrary elliptic complexes, for which one clearly needed other than pure algebraic geometry arguments. In retrospect I think here we took a small first step towards the sort of mixture of geometry, analysis and algebraic geometry found in the physics world whose influence on mathematics has been so great during the last twenty years.

Maybe it is therefore appropriate here, to explain at least my mind-set of that period, and to make a few elementary comments for the non-experts on Shimura's conjectures and our generalization. And, as is my wont, let me start at the beginning with a brief outline of what we might call the "Lefschetz principle" relating fixed points to traces of linear maps.

The simplest instance is that of a permutation $f: X \rightarrow X$ of a finite set. It is then clear that the number of diagonal entries in the permutation matrix of $f$ counts the number of fixed points in $X$ under $f$. The Lefschetz fixed point theorem deals with the topological situation where now $f$ can 
be any continuous map and $X$ is a compact space, say a finite complex.

Under these assumptions Lefschetz defines the Lefschetz number $L(f)$ of $f$ to be the alternating sum of the traces of the induced homomorphisms by $f$ in the cohomology $H^{*}(X)$ of $X$, and proves that when $L(f)$ is not 0 , then $f$ must have a fixed point. In the geometrically most transparent case one has a more precise result which has always been close to my heart, ever since I first learned of it in a paper of Heinz Hopf.

Namely, if $X$ is a smooth compact manifold and the graph of $f$ intersects the diagonal in $X \times X$ transversally, then $L(f)$ counts the "algebraic" number of fixed points of $f$. More precisely, each fixed point $p$ counts with the weight $c(p)$ given by the formula:

$$
c(p)=\operatorname{sign}\left(\operatorname{det}\left(1-f_{*, p}\right)\right),
$$

where $f_{*, p}$ denotes the derivative of $f$ at $p$ and det stands for determinant.

Thus the weight of a fixed point is + or -1 , and it is clearly this version which led André Weil to his famous conjectures concerning the counting of rational points on algebraic varieties over a finite field. In these conjectures $f$ was to be the Frobenius map, and the Lefschetz number $L(f)$ was taken to be an alternating sum of traces of an induced map by $f$ in a mythical cohomology theory. In 1964 these magnificent conjectures were still far from being proved, although some aspects of them had already been established.

It is strange, again in retrospect, that before 1964 no one had taken up the quite natural question of what the appropriate version of the above formula would be in the holomorphic or algebraic category. After all, Hirzebruch's amazing Riemann-Roch theorem had been with us for ten years by then, and his result can be thought of as a very special case of a Lefschetz formula: namely, as the evaluation of $L(f)$ with $f$ the identity map, but now acting on the sheaf cohomology of a nonsingular algebraic variety $X$. In short, from this point of view the Riemann-Roch question therefore dealt with $L(f)$ in the situation furthest removed from transversality.

I think it was this oversight then that explains Michael's and my delight at the formula Shimura had conjectured; to prove it seemed like a tailormade problem for some one with our interests. For Shimura's conjecture precisely filled this gap.

Indeed, for a transversal algebraic map $f$ of a nonsingular compact variety the Lefschetz number $L(f)$, taken with respect to the structure sheaf, was now conjectured to be a sum of contributions over the fixed 
points of $f$, with the weight of a fixed point $\mathrm{p}$ being:

$$
c(p)=\frac{1}{\operatorname{det}\left(1-f_{*, p}^{\prime}\right)},
$$

where $f_{*, p}^{\prime}$ denotes the holomorphic part of the derivative of $f$ at $p$.

\section{Remarks.}

(i) That these weights are now complex numbers was at first puzzling, but was soon found to be a manifestation of the fact that this cohomology obeyed no homotopy axiom.

(ii) The assumption of transversality is of course essential in all these formulas; however, in truly geometric situations they also carry the seed of the solution of the corresponding index problem, i.e., the evaluation of $L(f)$ when $f$ is the identity map. This occurs, for instance, in the Riemann-Roch situation if $X$ admits a holomorphic vector field $U$, with nondegenerate fixed point set. Exponentiating $U$, the transformations $\exp (t U)$ furnish one with a family of transversal maps approximating the identity and one is naturally led to study the limit of the fixed point formula as $t$ goes to 0 . The left-hand side then approaches the Riemann-Roch number and the right-hand side produces a sum over the fixed points $p$ of the constant term in the expansion of:

$$
\frac{1}{\operatorname{det}(1-\exp (-t u(p)))}
$$

where $u(p)$ denotes the infinitesimal action of $U$ on the tangent space of $X$ at $p$. In terms of the eigenvalues $\lambda_{1}, \ldots, \lambda_{n}$ of $u(p)$, one now easily finds this constant term to be:

$$
\operatorname{Todd}\left(\lambda_{1}, \ldots, \lambda_{n}\right) / \operatorname{det} u(p),
$$

where Todd denotes the Todd polynomial occurring in Hirzebruch's formula.

The beautiful formula (2) clearly made sense in the analytic category for a transversal holomorphic map and so was a first motivation to generalize it to other geometric situations. Eventually this generalization took the following form:

Suppose that $E^{*}$ denotes an elliptic complex over the compact manifold $X$. Let $f$ be a transversal map of $X$ into itself, and let $F$ denote a compatible lifting of $f$ to $E^{*}$. Note that this assumption implies that for 
each fixed point $p$ of $f$, the lifting $F$ induces an endomorphism $F(p)$ of $E^{*}$ restricted to $p$. We write $\ell(f, F ; p)$ for the alternating sum of the traces of $F(p)$, and $L(f, F)$ for the alternating sum of the traces of the map induced by the pair $(f, F)$ in the cohomology of $E^{*}$.

With this understood, our formula evaluates $L(f, F)$ as the sum of local contributions at the fixed points of $f$, each fixed point being counted with weight:

$$
c(p)=\frac{\ell(f, F ; p)}{\left|\operatorname{det}\left(1-f_{*, p}\right)\right|}
$$

Note that it is the absolute value of the determinant that enters in the denominator here and in this formula $f_{*, p}$ again denotes the derivative of $f$ at $p$.

I will leave it as an exercise for the reader to see that the relation (4) is compatible with the earlier ones (1) and (2). Indeed for the de Rham complex the numerator is seen to be the expansion of $\operatorname{det}\left(1-f_{*, p}\right)$ so that the ratio in question gives our first version. In the second instance the $\bar{\partial}$ operator contributes a factor to the numerator equal to the antiholomorphic part of $f_{*, p}$. Q.E.D.

The proof of this result is really quite simple. There are two salient points. The right-hand side is seen to be the product of two distributions with transversal wave front sets, and hence well defined. That this product descends to cohomology follows from the nonsingularity and transversality which enables one to approximate the distributions in a "flat manner".

One of the most exciting consequences of the extension from the holomorphic to the elliptic case was that in this realm these formulae became applicable to the Dirac operator and its twistings on spin manifolds, and so eventually led, for instance, to the Whitney-Taubes rigidity theorems. This in turn ushered in subjects such as elliptic cohomology. Possibly even more exiting are the applications of far-reaching generalizations of these fixed point formulae in the recent work on mirror symmetry and related topics.

I hope that this state of affairs will excuse my, possibly over-leisurely, exposition of their humble origins.

Finally let me end these reminiscences with my best wishes for the future of the Woods Hole meetings: may they prosper and thrive.

Raoul Bott

April 16, 2004 
This page is intentionally left blank 


\section{CONTENTS}

Preface v v

Introduction by R. Bott vii

Quantizing Teichmüller Spaces Using Graphs 1

L. Chekhov

Lectures on Indices and Relative Indices on Contact and CR-Manifolds

C. L. Epstein

Biologic II

L. H. Kauffman

Operads, Moduli of Surfaces and Quantum Algebras

R. M. Kauffmann

Fragments of Nonlinear Grothendieck-Teichmüller Theory

P. Lochak

Cell Decomposition and Compactification of Riemann's Moduli Space in Decorated Teichmüller Theory

R. C. Penner

Spatial Intermittency in Two-Dimensional Turbulence:

A Wavelet Approach

K. Schneider, M. Farge and N. Kevlahan

An Elementary Definition of Brownian Motion in Hilbert Space

N. Tongring 